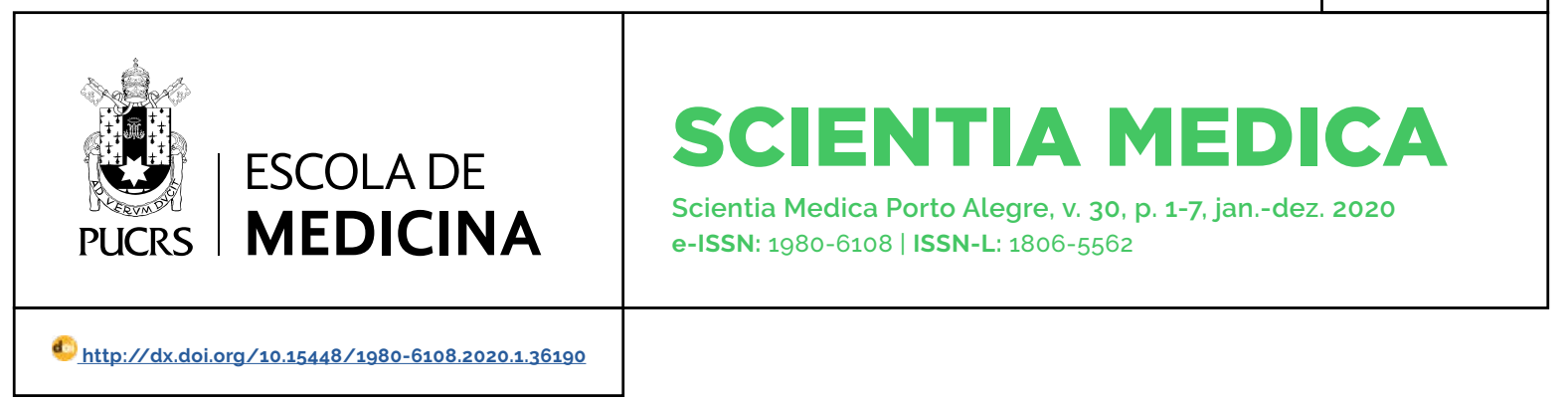

EDUCAÇÃO EM CIÊNCIAS DA SAÚDE

\title{
Análise das ligas acadêmicas de medicina sob a perspectiva dos alunos
}

\author{
Analysis of academic medical leagues from the students' perspective
}

\author{
Gustavo Bittencourt Camilo \\ orcid.org/0000-0001-7387-8381 \\ gustavoscamilo@hotmail.com
}

Marcus Gomes Bastos ${ }^{1}$

orcid.org/0000-0003-3651-9923

marcusbastos7@gmail.com

Gabriela Cumani Toledo ${ }^{1}$

orcid.org/0000-0003-2654-8894

gabrielacumanig@gmail.com

Ana Paula Ferreira ${ }^{1}$

orcid.org/ 0000-0002-5761-798X

paulaffisio@gmail.com

Tainá Gomes Brandão ${ }^{1}$

orcid.org/0000-0002-4045-5646

tainag.brandao@hotmail.com

Ana Flávia Miranda Reis ${ }^{1}$

orcid.org/0000-0003-1112-8301

a.flavinha@yahoo.com.br

Isabella de Almeida Paes

Barretto Coutinho ${ }^{1}$

orcid.org/0000-0003-4511-5576

isaapbc@gmail.com

Gabriel Lunardi Aranha ${ }^{1}$

orcid.org/0000-0003-2875-3189

gabriell.aranha@gmail.com

Bárbara Isadora Amâncio de Souza ${ }^{1}$

orcid.org/0000-0002-9203-5117

barbara.amancio@hotmail.com.br

Recebido em: 4/11/2019.

Aprovado em: $8 / 8 / 2020$.

Publicado em: 21/12/2020.

\section{(c) (1)}

Artigo está licenciado sob forma de uma licença Creative Commons Atribuição 4.0 Internacional.

\section{Resumo}

Objetivo: analisar as ligas acadêmicas de medicina (LAM) estruturadas e em funcionamento em instituição de ensino superior como estratégias de ensino e aprendizagem, considerando o perfil e verificar se as atividades promovidas pelas LAM estão alinhadas com a medicina baseada em evidências.

Métodos: estudo descritivo, do tipo transversal, aprovado pelo Comitê de Ética da Faculdade de Ciências Médicas e da Saúde de Juiz de Fora. Participaram como sujeitos da pesquisa estudantes do último ano de medicina e médicos recém-formados no ano de 2018. Essa amostragem não probabilística teve como finalidade a avaliação da influência das LAM na formação médica.

Resultados: oito (5.9\%) entrevistados não participaram de qualquer LAM. Observou-se que 31 (24,2\%), 49 (38,3\%) e 38 (29,7\%) participaram de uma, duas ou três LAM, respectivamente. A maioria participou de LAM do terceiro ao oitavo periodo, porém em todos os períodos houve participação de algum aluno. Para 50 (39,1\%) entrevistados, as LAM ajudaram de alguma forma para confirmar a escolha da área da residência, já para $53(41,4 \%)$ deles, elas ajudaram na exclusão da área para a residência. Nota-se, ainda, que $117(91,4 \%)$ dos que participaram de LAM acharam a experiência válida para a formação acadêmica.

Conclusões: a maior parte dos alunos atualmente participam de ligas; há enorme interesse em participar de diretoria de LAM e estágios extracurriculares; as LAM contribuem para a escolha da futura especialidade; os estudantes veem necessidade na regulamentação das LAM; a avaliação global é de que a experiência é válida para a formação médica. O tema ligas acadêmicas é de enorme relevância atualmente em virtude da extensa participação dos estudantes nessas atividades. A educação em saúde visa compreender a importância dessas entidades para garantir o maior êxito possivel no ensino, na pesquisa e na extensão.

Palavras-chave: educação médica, estudantes de medicina, ciências da saúde. Abstract

Aims: the objective was to analyze the structured and functioning of academic medical leagues (AML) in higher education institution as teaching and learning strategies, considering the profile and to verify if the activities promoted by the AML are aligned with the evidence-based medicine.

Methods: descriptive, cross-sectional study, approved by the Ethics Committee of the Faculty of Medical and Health Sciences of Juiz de Fora. Medical students and newly graduated physicians participated in the research in 2018. This non-probabilistic sampling aimed to evaluate the influence of AML on medical education. Results: eight respondents did not participate in AML (5.9\% of the sample). 31 (24.2\%), 49 (38.3\%) and 38 (29.7\%) participated in one, two or three AML, respectively. Most participated in AML from the third to the eighth period, but in all periods, there was participation. For 50 (39.1\%) of respondents, the AML helped in some way to confirm the choice of area of residence, while for $53(41.4 \%)$ of them, AML helped to exclude the area for residence. It is also noted that 117 (91.4\%) of those who participated in AML found the experience valid for academic education. 
Conclusions: thus, most students currently participate in leagues; There is tremendous interest in attending AML board and extracurricular internships; AML contribute to the choice of future specialty; Students needs AML regulation; The overall assessment is that experience is valid for medical education. The academic leagues theme is of enormous relevance today because of the extensive participation of students in these activities. Health education aims to understand the importance of these entities to ensure the greatest possible success in teaching, research, and extension.

Keywords: medical education, medical students, health sciences

ABREVIATURAS: LA, ligas acadêmicas; USP, Universidade de São Paulo; LAM, Liga acadêmica de medicina.

\section{Introdução}

As ligas acadêmicas de medicina (LAM) são entidades formadas por grupos de alunos de diferentes anos da graduação que possuem interesse por um determinado tema sob a supervisão de profissionais e de professores vinculados à instituição de ensino superior ou a hospitais de ensino $[1,2]$. Participam de forma efetiva na educação médica, promovendo conhecimento e atuação em áreas específicas não contempladas pelos currículos tradicionais, permitindo aproximação do estudante com as especialidades e, consequentemente, com o sistema de saúde [3,4].

De modo geral, as LAM incorporam e buscam a associação do ensino, da pesquisa e da extensão; assumem um caráter extracurricular e complementar; e suas ações são de natureza teórica e prática $[5,6,7]$. As atividades teóricas são desenvolvidas por meio de aulas, seminários, análise e discussão de textos, apresentações de casos clínicos e realização de eventos científicos. As atividades práticas são desenvolvidas em ambulatórios, hospitais, instituições filantrópicas filiadas e unidades básicas de saúde [3,5,8,9].

No Brasil, a primeira liga acadêmica de medicina foi fundada na década de 1920, na Faculdade de Medicina da Universidade de São Paulo (USP) [9]. Hoje em dia, o que se percebe é que o grande número de LAM de medicina existentes é fruto da necessidade, por parte do aluno, de ter um papel mais ativo na sua formação como médico, além de compensar as lacunas da grade curricular do modelo clássico [3,10,11,12,13]. Tendo como objetivo também, a melhora no currículo e o desejo de integrar-se a outros alunos que possuem os mesmos interesses por um determinado tema [14,15]. A participação em LAM são as principais atividades extracurriculares desempenhadas pelos estudantes durante o curso [16,17].

O objetivo deste estudo foi analisar as LAM estruturadas e em funcionamento na Faculdade de Ciências Médicas e da Saúde de Juiz de Fora (SUPREMA), como estratégias de ensino e de aprendizagem, considerando o perfil, e verificar se as atividades promovidas pelas LAM estão alinhadas com a medicina baseada em evidências. Devido ao relativo baixo número de trabalhos científicos na área, não se sabe ainda o impacto real delas na formação médica, sendo necessários novos estudos para uma melhor compreensão do tema.

\section{Métodos}

$O$ atual trabalho se classifica como descritivo do tipo transversal. O estudo foi aprovado pelo Comitê de Ética da Faculdade de Ciências Médicas e da Saúde de Juiz de Fora (SUPREMA), sob o parecer consubstanciado $n^{\circ} 2.250 .093$. Todos os participantes leram e assinaram o Termo de Consentimento Livre e Esclarecido.

Participaram como sujeitos da pesquisa, estudantes do último ano de medicina e médicos recém-formados no ano de 2018 da Faculdade de Ciências Médicas e da Saúde de Juiz de Fora. Essa amostragem não probabilistica teve como finalidade a avaliação da influência das LAM na formação médica.

A coleta de dados foi realizada mediante entrevistas simples. O instrumento utilizado foi um questionário estruturado com perguntas fechadas, previamente testado contendo 24 perguntas. Foram abordados os seguintes aspectos sobre as LAM: a) participação em ligas acadêmicas; b) atuação em diretoria de ligas acadêmicas; c) realização de estágios e associação com a liga; d) percepção de ajuda na escolha da especialidade através das ligas; e) contribuição das ligas na formação médica; f) motivação para participar das ligas; e g) necessidade de regulação das ligas. Os dados foram analisados descritivamente por meio de tabelas de distribuição de frequências absolutas e relativas. 


\section{Resultados}

No total foram entrevistados 136 formandos e médicos residentes, dos quais 128 (94,1\%) participaram de alguma LAM. Oito (5.9\%) entrevistados não participaram de LAM e justificaram que preferiram se dedicar a outras atividades. Observa-se, ainda, que a maioria participou de LAM do terceiro ao oitavo periodo, porém em todos os períodos houve participação (Tabela 1).

TABELA 1 - Organização das LAM e motivações a não participar das mesmas $(n=128)$

\begin{tabular}{|c|c|c|}
\hline Variável & Respostas & Frequência (\%) \\
\hline \multirow[t]{5}{*}{ Número de ligas em que } & 1 & $31(24,2)$ \\
\hline & 2 & $49(38,3)$ \\
\hline & 3 & $38(29,7)$ \\
\hline & 4 & $9(7,0)$ \\
\hline & 5 & $1(0,8)$ \\
\hline \multirow{12}{*}{$\begin{array}{l}\text { Qual foi o periodo } \\
\text { da faculdade participou?* }\end{array}$} & 1 & $1(0,8)$ \\
\hline & 2 & $12(9,4)$ \\
\hline & 3 & $18(14,1)$ \\
\hline & 4 & $32(25,0)$ \\
\hline & 5 & $40(31,2)$ \\
\hline & 6 & $45(35,2)$ \\
\hline & 7 & $32(25,0)$ \\
\hline & 8 & $24(18,8)$ \\
\hline & 9 & $11(8,6)$ \\
\hline & 10 & $11(8,6)$ \\
\hline & Preferiu outras áreas para não haver especialização precoce & $11(8,5)$ \\
\hline & Não havia escolhido a especialidade & $25(19,5)$ \\
\hline \multirow{5}{*}{$\begin{array}{l}\text { Qual foi o motivo de não } \\
\text { ter participado de alguma } \\
\text { LAM afim de sua área de } \\
\text { especialização* }\end{array}$} & Não conseguiu passar na área de interesse & $9(7,0)$ \\
\hline & Existiam outras ligas com melhores atividades práticas & $14(10,9)$ \\
\hline & Existiam outras ligas com melhores atividades acadêmicas & $4(3,1)$ \\
\hline & Não respondeu & $1(0,7)$ \\
\hline & Não participou de ligas de sua especialidade & $64(50)$ \\
\hline
\end{tabular}

LAM, Ligas Acadêmicas de Medicina. "Pode haver mais de uma resposta para cada individuo.

Verificam-se, ainda, que 91 (71,1\%) dos participantes foram diretores, 88 (68,8\%) fizeram estágio extracurricular vinculado a alguma LAM e 67 (52,3\%) fizeram parte de LAM afim de sua área de especialização. Além disso, do total de entrevistados, $90(70,6 \%)$ fizeram estágio extracurricular oficial não vinculado às LAM, apesar de $77(60,4 \%)$ deles terem respondido que havia LAM deste tema na faculdade.

Os alunos têm forte interesse na regulamentação das LAM. A melhora no conhecimento sobre a área da LAM é apontada como a principal contribuição das LAM. (Tabela 2). 
TABELA 2 - Importância na escolha de residência, formação e regulamentação $(n=128)$

Variável

Respostas

Frequência (\%)

\begin{tabular}{|c|c|c|}
\hline \multirow{3}{*}{$\begin{array}{l}\text { As LAM ajudaram de alguma forma na } \\
\text { escolha de sua residência? }\end{array}$} & Sim, para exclusão & $53(41,4)$ \\
\hline & Sim, para confirmação & $50(39,1)$ \\
\hline & Não & $25(19,5)$ \\
\hline \multirow{4}{*}{$\begin{array}{l}\text { Acha a experiência válida para a formação? } \\
\text { Acha necessária a regulamentação de } \\
\text { LAM por algum órgão? }\end{array}$} & Sim & $117(91,4)$ \\
\hline & & \\
\hline & Sim & $108(84,0)$ \\
\hline & Melhora no conhecimento sobre a área & $97(75,8)$ \\
\hline \multirow[t]{5}{*}{ Contribuição para a formação médica* } & $\begin{array}{l}\text { Déficit da faculdade na cadeira da LAM, } \\
\text { melhorando conhecimento }\end{array}$ & $32(25,0)$ \\
\hline & Maior interação com acadêmicos e professores & $26(20,3)$ \\
\hline & Aumento da motivação no curso & $25(19,5)$ \\
\hline & Contato com atividades de pesquisa & $14(10,9)$ \\
\hline & Nenhuma & $19(14,8)$ \\
\hline
\end{tabular}

LAM, Ligas Acadêmicas de Medicina. "Pode haver mais de uma resposta para cada indivíduo.

O enriquecimento do currículo e a possibilidade de aproximação com uma área afim foram as principais motivações dos estudantes para par- ticipação nas LAM. O pouco comprometimento dos participantes e dos orientadores foram as principais falhas apontadas das LAM (Tabela $\mathbf{3}$ ).

TABELA 3 - Motivações, falhas e resultados após a realização de LAM $(n=128)$

\begin{tabular}{|c|c|c|}
\hline Variável & Respostas* & Frequência (\%) \\
\hline \multirow[t]{8}{*}{ Motivo de ter feito LAM } & Enriquecer o currículo & $107(83,6)$ \\
\hline & Se aproximar de uma área afim & $83(64,8)$ \\
\hline & Devido à pressão do meio & $40(31,2)$ \\
\hline & Insuficiência curricular na área & $34(26,6)$ \\
\hline & Professor orientador & $10(7,8)$ \\
\hline & Nenhuma das anteriores & $4(3,1)$ \\
\hline & Pouco comprometimento do orientador & $73(57,0)$ \\
\hline & Pouco comprometimento dos participantes & $72(56,2)$ \\
\hline \multirow{6}{*}{ Falhas da LAM } & Poucas atividades & $52(40,6)$ \\
\hline & Conhecimento muito específico & $11(8,6)$ \\
\hline & Excesso de atividades & $10(7,8)$ \\
\hline & Nenhuma falha & $48(37.5)$ \\
\hline & Outras & $1(0,8)$ \\
\hline & Conhecimento da rotina do profissional & $64(50,0)$ \\
\hline \multirow[t]{3}{*}{ Colaboração para a escolha final ${ }^{* *}$} & Primeiro contato com a prática médica & $53(41,4)$ \\
\hline & Conhecimento da qualidade do profissional & $48(37,5)$ \\
\hline & Conhecimentos teóricos específicos da área & $46(35.9)$ \\
\hline
\end{tabular}

LAM, Ligas Acadêmicas de Medicina. "Pode haver mais de uma resposta para cada indivíduo. ** 22 participantes não responderam este questionamento. 


\section{Discussão}

O primeiro dado objetivo que nos chama a atenção é a de que a imensa maioria dos estudantes do último ano participou de alguma LAM. Tal fato é particularmente intuitivo a quem acompanha o cotidiano dos estudantes atualmente, havendo vagas suficientes para todos os alunos [18]. Isso corrobora o estudo de Peres et al. que afirmou que cerca de $70-90 \%$ dos alunos haviam participado de ligas na USP de Ribeirão Preto, destacando-se que, como nosso estudo foi realizado cerca de 12 anos após o citado, há ainda maior participação dos estudantes em LAM, já superior a 94\% em nossa instituição [6].

Quanto à presença em diretorias de ligas e em estágios extracurriculares, destacam-se dois aspectos: o interesse em organizar a própria entidade e, consequentemente, eventos científicos e simpósios, bem como a necessidade de organizar institucionalmente essas entidades, havendo inclusive guias para sugestões de formação de ligas $[18,19]$. Toledo et al., já havia destacado a preocupação dos preceptores em regulamentar as LAM, o que determina maior comprometimento entre os integrantes das mesmas [3]. O estudo de Yang et al., a respeito de liga de Anatomia deu ênfase à possibilidade de os estudantes aprimorarem habilidades administrativas e burocráticas nas LAM, valorizando a necessidade de organização técnica dessas entidades [20].

Outro dado de extrema importância é de que mais de $80 \%$ dos alunos acreditam que sua participação ajudou a escolher ou excluir essa opção para a futura especialidade médica. Ferreira et al. [15] destacaram que ao longo da passagem dos alunos pela liga há importante crescimento no interesse em cursar futuramente a especialidade de cirurgia plástica, demonstrando inclusive dados numéricos com relevância estatística [15]. Da mesma forma, Ramalho et al. [13], observaram ganho em conhecimento técnico e de informações a respeito da anestesiologia com evolução objetiva em testes cognitivos.

Destaca-se, ainda, que quase $30 \%$ das escolhas do aluno para qual liga participar perpassam por interesses em atividades de pesquisa e impor- tância prática, indicando que eles desejam mais do que tão somente a pontuação advinda das LAM. Moreira et al. [18], em análise qualitativa, foram veementes ao demonstrar que os alunos têm necessidade de buscar experiência como profissional médico e de serem responsáveis pelo acompanhamento longitudinal dos pacientes contrastando ao tradicional currículo essencialmente teórico [18]. Kara Jose et al. [21] demonstraram que grande parte das ligas de oftalmologia oferecia atividades de pesquisa e de extensão, o que era extremamente apreciado pelos estudantes.

Notável constatar o interesse e a preocupação na pontuação de créditos para o currículo da própria instituição e para as provas de residência médica. Nosso estudo demonstrou que $83,6 \%$ dos alunos almejam melhora dos currículos visando futuros concursos de residência, indo ao encontro ao estudo de Chaves et al. [18] que mostrava que $54 \%$ das provas na região Sudeste valorizavam as ligas. Algumas faculdades, como a Unifesp, exigem quantidade mínima de créditos e as ligas são capazes de fornecer pontuação nesse sentido.

Deve-se discutir, também, as principais falhas e incongruências das LAM. Em nosso estudo, número superior a $55 \%$ dos estudantes relatam que a falta de comprometimento de alunos e de preceptores são as principais falhas das LAM da instituição, assemelhando-se ao já referido em estudo anterior, que cita a ausência de alguns preceptores que delegam a função para médicos residentes e recém-formados [18]. Do mesmo modo, escritos sobre a análise dos orientadores reforçam a discussão de que, muitas vezes, a preocupação dos estudantes é muitas vezes mais enfática para adquirir curriculo do que propriamente conhecimento [3].

É premente ressaltar que um número superior a $90 \%$ é favorável às LAM em contexto global. A aproximação com atividades práticas, maior contato com profissionais da área e a discussão científica ou de casos clinicos é importante para a formação dos estudantes, bem como para a escolha da futura especialidade conforme foram citados em muitos outros trabalhos $[2,3,4,7,12,14,16,18,19]$. 
Finalmente, os achados principais desse estudo foram os seguintes: a maior parte dos alunos atualmente participa de ligas; há enorme interesse em participar de diretoria de LAM e de estágios extracurriculares; as LAM contribuem para a escolha da futura especialidade; os estudantes veem necessidade na regulamentação das LAM; a avaliação global é de que a experiência é válida para a formação médica.

\section{Notas}

\section{Apoio financeiro}

Este estudo não recebeu apoio financeiro de fontes externas.

\section{Declaração de conflito de interesses}

Os autores declaram não haver conflitos de interesses relevantes ao conteúdo deste estudo.

\section{Contribuições dos autores}

Todos os autores fizeram contribuições substanciais para concepção, ou delineamento, ou aquisição, ou análise ou interpretação de dados; e redação do trabalho ou revisão crítica; e aprovação final da versão para publicação.

\section{Disponibilidade dos dados e responsabilidade pelos resultados}

Todos os autores declaram ter tido total acesso aos dados obtidos e assumem completa responsabilidade pela integridade destes resultados.

\section{Referências}

1- Cavalcante ASP, Vasconcelos MIO, Lira GV, Henriques RLM, Albuquerque INM, Maciel GP, Ribeiro MA, Gomes DF. As Ligas Acadêmicas na Área da Saúde: Lacunas do Conhecimento na Produção Científica Brasileira. Rev Bras Educ Med. 2018;42(1):197-204. https://doi. org/10.1590/1981-52712018v42n1rb20170081

2- Bastos, MLS, Trajman A, Teixeira EG, Selig L, Belo MTCT. The role of academic associations in professional training. J Bras Pneumol. 2012;38(6):803-5. https://doi. org/10.1590/S1806-37132012000600018

3- Toledo GC, Bastos MG, Barbosa KM, Araújo PC, Aranha GL, Ferreira AP, Camilo, GB. Ligas acadêmicas na educação médica: uma análise institucional sob a visão dos orientadores. HU Revista. 2020;45(4):421-5. https://doi.org/10.34019/1982-8047.2019.v45.27899
4- Imakuma ES. As ligas acadêmicas no Ensino Médico. Rev Med. 2013:92(4):271-2. https://doi.org/10.11606/ issn.1679-9836.v92i4p271-272

5- Pego-Fernandes PM, Mariani AW. O ensino médico além da graduação: ligas acadêmicas. Diagn Tratamento. 2011;16(2):50-1.

6- Santana, AGDA. Academic student leagues. The merit and reality. Medicina (Ribeirão Preto). 2012;45(1):96-8. https://doi.org/10.11606/issn.2176-7262.v45i1pg6-98

7- Peres, CM, Andrade AS, Garcia SB. Extracurricular Activities: Multiplicity and Differentiation Required for the Curriculum. Rev Bras Educ Med. 2007:31(3):203-11. https://doi.org/10.1590/S0100-55022007000300002

8- Belei RA, Gimeniz-Paschoal SR, Nascimento EN. História Curricular dos Cursos de Graduação da Área da Saúde. História da Educação. 2008;12(24):101-120.

9- Hamamoto Filho PT. Ligas acadêmicas: motivações e críticas a propósito de um repensar necessário. Rev Bras Educ Med. 2011:35(4):535-43. https://doi.org/10.1590/ $\underline{\text { S0100-55022011000400013 }}$

10- Queiroz SJ, Azevedo RLO, Lima KP, Lemes MMDD, Andrade M. A importância das ligas acadêmicas na formação profissional e promoção de saúde. Fragmentos de cultura. 2014:24:73-8.

11- Torres AR, Oliveira GM, Yamamoto FM, Lima MCP. Academic Leagues and medical education: contributions and challenges. Interface. 2008;12(27):713-20. https://doi.org/10.1590/S1414-32832008000400003

12- Hamamoto Filho PT, Venditti VC, Miguel L, Silva LA, Oliveira CC. Peraçoli, JC. Pesquisa em Educação Médica conduzida por Estudantes: um Ano de Experiência do Núcleo Acadêmico de Pesquisa em Educação Médica. Rev Bras Educ Med. 2011;35(1):108-113. https://doi. org/10.1590/S0100-55022011000100015

13- Ramalho AS, Silva FD, Kronemberger TB, Pose RA, Torres MLA, Carmona MJC, Auler JR JOC. Ensino de Anestesiologia durante a Graduação por meio de uma Liga Acadêmica: qual o Impacto no Aprendizado dos Alunos? Rev Bras Anestesiol. 2012;62(1):63-73. https:// doi.org/10.1590/S0034-70942012000100009

14- Silva AS, Flores O. Ligas Acadêmicas no Processo de Formação dos Estudantes. Rev Bras Educ Med. 2015:39(3):410-25. https://doi.org/10.1590/ 1981-52712015v39n3e02592013

15- Ferreira LL, Monteiro M, Cunha MS, Oliveira WL, Bandeira NG, Menezes JV. Ligas Acadêmicas: o que há de positivo? Experiência de implantação da Liga de Cirurgia Plástica. Rev Bras Cir Plást. 2008:23(3):158-61.

16- Hamamoto Filho PT, Villas-Bôas PJF, Corrêa FG, Munõz GOC, Zaba M, Venditti VC, Schellini SA. Regulation of student leagues: the experience at the Botucatu School of Medicine. Rev Bras Educ Med. 2010;34(1):160-7. https://doi.org/10.1590/S0100-55022010000100019 
17- Botelho NM, Ferreira IG, Souza LEA. Medicine academic leagues: review article. Revista Paraense de Medicina. 2013:27(4):85-8.

18- Moreira LM, Mennin RHP, Lacaz, FAC, B VC. Ligas Acadêmicas e Formação Médica: Estudo Exploratório numa Tradicional Escola de Medicina. Rev Bras Educ Med. 2019;43(1):115-125. https://doi.org/10.15.90/ 1981-52712015V43n1rb20170141

19- Azevedo RP, Dini PS. Guia para construção de Ligas Acadêmicas. Assessoria Científica da Direção Executiva Nacional dos Estudantes de Medicina, 2006.

20- Yang GYH, Braga ACB, Hipólito NC, Vieira KST, Pessanha CG, Abrantes FG, Pereira OS, Corrêa CL. Liga de Anatomia Aplicada (LAA): as Múltiplas Perspectivas sobre Participar de uma Liga Acadêmica. Rev Bras Educ Med. 2019:43(1):80-6. https://doi.org/10.1590/ 1981-52712015v43n1rb20170146

21- Kara José AC, Passos LB, Kara José FC, Kara José $\mathrm{N}$. Ensino extracurricular em Oftalmologia: grupos de estudos/ligas de alunos de graduação. Rev Bras Educ Med. 2007:31(2):166-72. https://doi.org/10.1590/ $\underline{\mathrm{S} 0100-55022007000200007}$

\section{Gustavo Bittencourt Camilo}

Graduado em Medicina pela Universidade Federal de Juiz de Fora (UFJF), em Juiz de Fora, MG. Mestre e Doutor em Ciências Médicas pela Universidade do Estado do Rio de Janeiro (UERJ), no Rio de Janeiro, RJ. Professor de Anatomia e Radiologia na Faculdade de Ciências Médicas e da Saúde de Juiz de Fora (SUPREMA), em Juiz de Fora, MG.

\section{Marcus Gomes Bastos}

Doutor em Medicina (Nefrologia) pela Universidade Federal de São Paulo (UNIFESP), em São Paulo, SP. Professor Adjunto IV de Nefrologia pela Universidade Federal de Juiz de Fora (UFJF), Juiz de Fora, MG; professor da Faculdade de Ciências Médicas e da Saúde de Juiz de Fora (SUPREMA, em Juiz de Fora, MG); graduado em Medicina pela Universidade Federal de Juiz de Fora (UFJF, Juiz de Fora, MG). Mestre em Ciências Médicas pela Universidade do Estado do Rio de Janeiro (UERJ), no Rio de Janeiro, RJ.

\section{Gabriela Cumani Toledo}

Mestre com ênfase em educação médica pelo Programa de Pós-Graduação da Universidade Federal de Juiz de Fora (UFJF), em Juiz de Fora, MG. Graduada em Medicina pela Faculdade de Ciências Médicas e da Saúde de Juiz de Fora (SUPREMA), em Juiz de Fora, MG; Residência Médica em radiologia pelo Hospital Federal dos Servidores do Estado do Rio de Janeiro (HFSE).

\section{Ana Paula Ferreira}

Graduada em Fisioterapia pela Faculdade de Ciências Médicas e da Saúde de Juiz de Fora (SUPREMA), em Juiz de Fora, MG, Mestre pela UERJ e doutoranda em Ciências da Reabilitação pela Universidade Federal de Minas Gerais (UFMG), em Belo Horizonte, MG. Docente na Faculdade de Ciências Médicas e da Saúde de Juiz de Fora (SUPREMA), em Juiz de Fora, MG; e supervisora do estágio de Fisioterapia Cardiovascular no Hospital e Maternidade Therezinha de Jesus (HMTJ, Juiz de Fora, MG.

\section{Tainá Gomes Brandão}

Acadêmica de Medicina pela Faculdade de Ciências Médicas e da Saúde de Juiz de Fora (SUPREMA), em Juiz de Fora, MG.

\section{Ana Flávia Miranda Reis}

Acadêmica de Medicina pela Faculdade de Ciências Médicas e da Saúde de Juiz de Fora (SUPREMA), em Juiz de Fora, MG.

\section{Isabella de Almeida Paes Barretto \\ Coutinho}

Acadêmica de Medicina pela Faculdade de Ciências Médicas e da Saúde de Juiz de Fora (SUPREMA), em Juiz de Fora, MG

\section{Gabriel Lunardi Aranha}

Graduação em Medicina pela Faculdade de Ciências Médicas e da Saúde de Juiz de Fora (SUPREMA), em Juiz de Fora, MG. Residente de Cirurgia Geral do Hospital Israelita Albert Einstein, em São Paulo, SP, Brasil. Médico do Hospital Naval Marcilio Dias, no Rio de Janeiro, RJ, Brasil.

\section{Bárbara Isadora Amâncio de Souza}

Acadêmica de Medicina pela Faculdade de Ciências Médicas e da Saúde de Juiz de Fora (SUPREMA), em Juiz de Fora, MG.

\section{Endereço para correspondência}

Gustavo Bittencourt Camilo

Alameda Salvaterra, 200,

Bairro Salvaterra, 36.033003

Juiz de Fora, MG, Brasil 